\title{
An experimental test of how parasites of predators can influence trophic cascades and ecosystem functioning
}

\author{
Jaime M. Anaya-Rojas, ${ }^{1,2,9}$ Rebecca J. Best, ${ }^{2,3}$ Franziska S. Brunner,,${ }^{4,5}$ Christophe Eizaguirre, ${ }^{5}$ \\ Miguel Costa Leal,${ }^{6,7}$ Carlos J. Melián, ${ }^{7}$ Ole Seehausen, ${ }^{7,8}$ and Blake Matthews ${ }^{2}$ \\ ${ }^{1}$ Department of Biological Science, Florida State University, Tallahassee, Florida 32306 USA \\ ${ }^{2}$ Center for Evolution \& Biogeochemistry, Eawag, Swiss Federal Institute for Aquatic Science and Technology, Aquatic Ecology \\ Seestrasse 79, Kastanienbaum 6047 Switzerland \\ ${ }^{3}$ School of Earth and Sustainability, Northern Arizona University, 525 South Beaver Street, Flagstaff, Arizona 86011 USA \\ ${ }^{4}$ Institute of Integrative Biology, University of Liverpool, Liverpool L69 3BX UK \\ ${ }^{5}$ School of Biological and Chemical Sciences, Queen Mary University of London, Mile End Road, London E1 4NS UK \\ ${ }^{6}$ MARE - Marine and Environmental Sciences Centre, Faculdade de Ciências da Universidade de Lisboa, Campo Grande, Lisboa \\ 1749-016 Portugal \\ ${ }^{7}$ Fish Ecology and Evolution Department, Center for Evolution \& Biogeochemistry, Eawag, Swiss Federal Institute for Aquatic Science \\ and Technology, Seestrasse 79, Kastanienbaum 6047 Switzerland \\ ${ }^{8}$ Institute of Ecology \& Evolution, Aquatic Ecology \& Evolution, University of Bern, Baltzerstrasse 6, Bern 3012 Switzerland
}

Citation: Anaya-Rojas, J. M., R. J. Best, F. S. Brunner, C. Eizaguirre, M. C. Leal, C. J. Melián, O. Seehausen, and B. Matthews. 2019. An experimental test of how parasites of predators can influence trophic cascades and ecosystem functioning. Ecology 100(8):e02744. 10.1002/ecy. 2744

Abstract. Parasites can shape the structure and function of ecosystems by influencing both the density and traits of their hosts. Such changes in ecosystems are particularly likely when the host is a predator that mediates the dynamics of trophic cascades. Here, we experimentally tested how parasite load of a small predatory fish, the threespine stickleback, can affect the occurrence and strength of trophic cascades and ecosystem functioning. In a factorial mesocosm experiment, we manipulated the density of stickleback (low vs. high), and the level of parasite load (natural vs. reduced). In addition, we used two stickleback populations from different lineages: an eastern European lineage with a more pelagic phenotype (Lake Constance) and a western European lineage with a more benthic phenotype (Lake Geneva). We found that stickleback caused trophic cascades in the pelagic but not the benthic food chain. Evidence for pelagic trophic cascades was stronger in treatments where parasite load of stickleback was reduced with an antihelmintic medication, and where fish originated from Lake Constance (i.e., the more pelagic lineage). A structural equation model revealed that differences in stickleback lineage and parasite load were most likely to impact trophic cascades via changes in the composition, rather than overall biomass, of zooplankton communities. Overall, our results provide experimental evidence that parasites of predators can influence the cascading effects of fish on lower trophic levels with consequences on ecosystem functioning.

Key words: density-mediated interactions; ecosystem functioning; mesocosms; parasites; stickleback; structural equation models; trait-mediated interactions; trophic cascades.

\section{INTRODUCTION}

In a classic trophic cascade, predators alter the biomass and/or community structure (species biomass and composition) of lower trophic levels and even ecosystem functioning (e.g., gross primary productivity and decomposition) via a combination of direct and indirect ecological effects (Polis et al. 2000, Ripple et al. 2016). In food chains composed of predators, grazers, and primary producers, predators can increase the biomass of primary producers by decreasing the density or biomass

Manuscript received 4 July 2018; revised 21 March 2019; accepted 29 March 2019. Corresponding Editor: Evan L. Preisser.

${ }^{9}$ E-mail: janaya@fsu.edu of grazers (density-mediated indirect effects or DMIE, Fig. 1a; Abrams 1995). Alternatively, predators can change the behavior of grazers, for example by reducing their activity and feeding rates, and thereby increase the biomass of primary producers via trait-mediated indirect effects (TMIE; Fig. 1b; Abrams 1995, 2007). In addition, predators might shift the composition of grazer communities and thereby affect primary producers (Schmitz 2006).

Previous work on trophic cascades has shown that intraspecific variation in morphology, activity levels, consumption rates, or hunting strategies can have profound effects on the occurrence and strength of trophic cascades (Borer et al. 2005, Abrams 2007, Post et al. 2008, Schmitz 2008, Rudman et al. 2016, Start and 
(a)

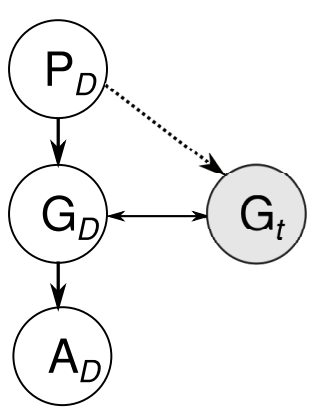

(b)

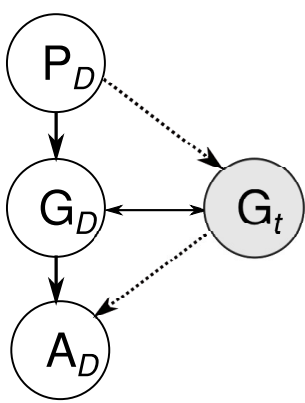

(c)

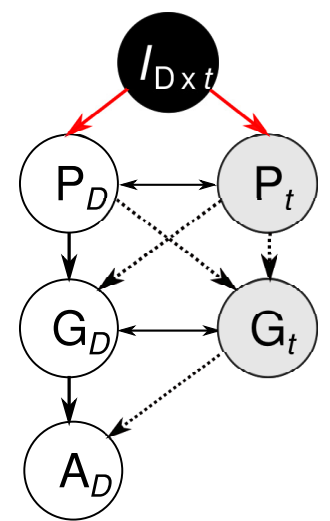

FIG. 1. Examples of trophic cascades in food webs with three trophic levels (a, b) and four trophic levels (c). Diagrams (a) and (b) represent food chains in which an algae population or community $(A)$ is eaten by a grazer population or community $(G)$, which is eaten by a predator population (P). Diagram (c) represents a food chain in which the predator population $(\mathrm{P})$ is infected by a parasite (I). Gray circles on the right-hand sides denote trait values or trait distributions influencing the species interaction $\left(G_{t}\right.$, a predator avoidance trait; or $P_{t}$, a predator foraging trait that is influenced by a parasite infection), while white circles represent variation in density (D) for any given trophic level (e.g., changes in predator density, PD). Solid and dotted arrows represent direct density and trait effects. Red arrows highlight the effects of parasite density and traits on predators. In food chain (a) there is a significant trophic cascade through density-mediated indirect effects (DMIE) but not DMIE on the algae population, unlike food chains (b) and (c). In food chain (c), parasite infections have significant trophic cascading effects on grazer and algae population.

Gilbert 2017), and many of these traits can be directly modified by parasite infections (Hatcher et al. 2006, Lafferty 2006, Lafferty and Kuris 2012). Parasites of predators can have trophic cascading effects both by reducing the population densities of their hosts (DMIE) and by changing host traits such as foraging activity and performance (TMIE; Fig. 1c), but the interaction of such parasite and predator effects have so far received little attention (Lefèvre et al. 2009, Hatcher and Dunn 2011). Previous research in aquatic systems has tested how parasites of grazers (e.g., Daphnia) can mediate the strength of trophic cascades by changing grazer foraging behavior (Duffy 2007, Wood and Johnson 2015), but we still lack evidence on whether and how parasites of predators affect the occurrence and strength of trophic cascades.

Whether parasites impact trophic cascades by changing predator density or changing predator behavior, the magnitude of their impacts likely depends on the co-evolutionary history between parasites and their hosts (Eizaguirre et al. 2012) as well as the host trophic position (Lafferty 2006). For instance, the evolution of host resistance to parasites can determine how strongly parasites affect host population density (DMIE; Hudson et al. 1998), as well as host condition and behavior for a given density (Anaya-Rojas et al. 2016, Buck and Ripple 2017). So far, studies have addressed how variation in ecological conditions (e.g., resources or temperature) affect the dynamics of host-parasite interactions (Wolinska and King 2009, Duffy et al. 2012).

To test whether parasites can influence variation in predator-mediated effects on ecosystem functioning in general, and on trophic cascades in particular, we performed a mesocosm experiment where we manipulated the density and parasite load of threespine stickleback
(Gasterosteus aculeatus). We were interested in addressing three main questions: (1) How do different parasite loads influence the direct and indirect ecological effects of stickleback? (2) What ecological pathways are most likely to underlie these effects? (3) How do stickleback lineages vary in their response to parasite load in a way that might impact their ecosystem effects?

In natural populations, stickleback are host to multiple parasite species that can impact their behavior and therefore their trophic interactions (Barber 2013). For instance, parasites such as Apatemon spp. and Diplostomum spp. can reduce stickleback consumption rates by reducing their vision (Seppälä et al. 2011). Intestinal parasites such as the cestode Schistocephalus solidus can actively manipulate stickleback feeding behavior (Milinski 1984), whereas ecotoparasites such as Gyrodactylus gasterostei can indirectly affect stickleback feeding behavior (Anaya-Rojas et al. 2016) and reduce body condition (Eizaguirre et al. 2009). In a previous mesocosm experiment, experimental infections of stickleback with $G$. gasterostei reduced stickleback body condition, thereby changing the effect of stickleback on both community composition and ecosystem functioning (Brunner et al. 2017). However, natural populations of stickleback are exposed to a large diversity of parasites (Eizaguirre et al. 2011), resulting in different co-evolutionary dynamics across fish populations (Eizaguirre et al. 2012). Hence, instead of manipulating individual parasite species (Brunner et al. 2017), we experimentally reduced parasite load by medicating stickleback with an antihelminth treatment. We used structural equation models to tease apart the direct and indirect effects of stickleback's parasite load and density on a broad range of ecosystem properties, including the total biomass and composition of the grazer community, the 
biomass of primary producers, and ecosystem functioning measured using an index of ecosystem multifunctionality (EMF; Byrnes et al. 2014).

The co-evolutionary history of stickleback populations with their parasite community might also influence how parasite load affects stickleback-mediated trophic cascades. In our experiment, we used stickleback from two European lineages that have recently colonized Switzerland: an eastern European lineage, represented by the population in Lake Constance, and a western European lineage, represented by the population in Lake Geneva (Lucek et al. 2010, Best et al. 2017, Fang et al. 2018). Previous work has shown that stickleback from the Lake Constance population have a more pelagic phenotype and feed more efficiently on pelagic prey than stickleback from the Lake Geneva population (Lucek et al. 2010, Best et al. 2017). In addition, these two populations have different parasite communities (Karvonen et al. 2015). We hypothesized that such lineage-specific differences might influence variation in the strength of sticklebackmediated trophic cascades in mesocosm ecosystems.

\section{Materials And Methods}

\section{Mesocosm experiment}

We conducted a large-scale mesocosm experiment with 45 outdoor cattle tanks $(1,000 \mathrm{~L}$ each) from 12 May to 14 June 2014 at the Center for Ecology, Evolution and Biogeochemistry of Eawag (Swiss Federal Institute of Aquatic Science and Technology) in Kastanienbaum, Switzerland. We collected male sticklebacks at the shores of Lake Constance $\left(47^{\circ} 29^{\prime} 56.6^{\prime \prime} \mathrm{N}\right.$, $\left.9^{\circ} 33^{\prime} 26.6^{\prime \prime} \mathrm{E}\right)$ and Lake Geneva (46 $23^{\prime} 53^{\prime \prime} \mathrm{N}, 6^{\circ} 53^{\prime} 08.6^{\prime \prime}$ E) with minnow traps placed overnight the first week of April 2014. All fish were kept in aquaria and fed with chironomids until the start of the experiment. In a complete randomized block design (Fig. 2a), replicated five times, we crossed eight factorial combinations of stickleback lineage (Constance or Geneva), two levels of fish densities (low $=3$ fish or high $=10$ fish, LD and HD, respectively), and parasite load (natural or reduced, $+I$ and $-I$ respectively), plus a control tank (no fish added). Stickleback densities used in this experiment are biologically relevant for this type of experiment (Matthews et al. 2016, Best et al. 2017, Brunner et al. 2017). During the experiment, dead fish were replaced with fish of similar size and from the same treatment combination to keep fish density constant during the experiment and avoid confounding effects of fish mortality.

\section{Manipulating parasite load}

Prior to the mesocosm experiment, we conducted a laboratory experiment to test the effectiveness of praziquantel (Koi MED ${ }^{\circledR}$ Worm-Ex ${ }^{\circledR}$, hereafter, Wormex) in reducing the parasite load of stickleback (36 fish in total: 18 from each lineage). Wormex is a commercial (a)

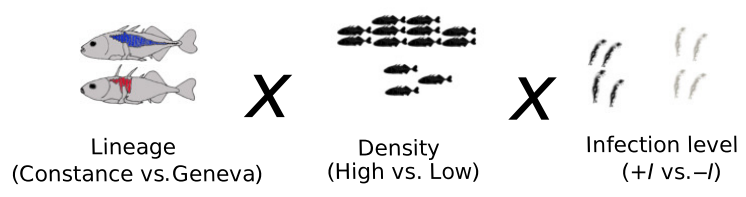

(b)

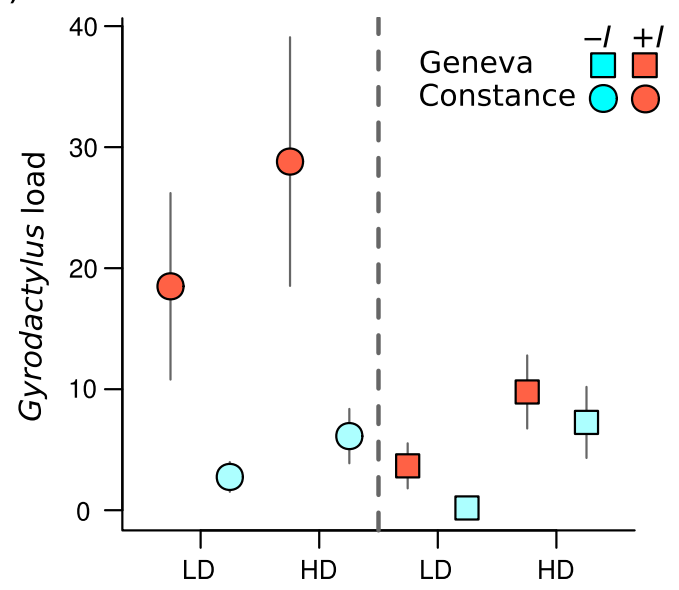

(c)

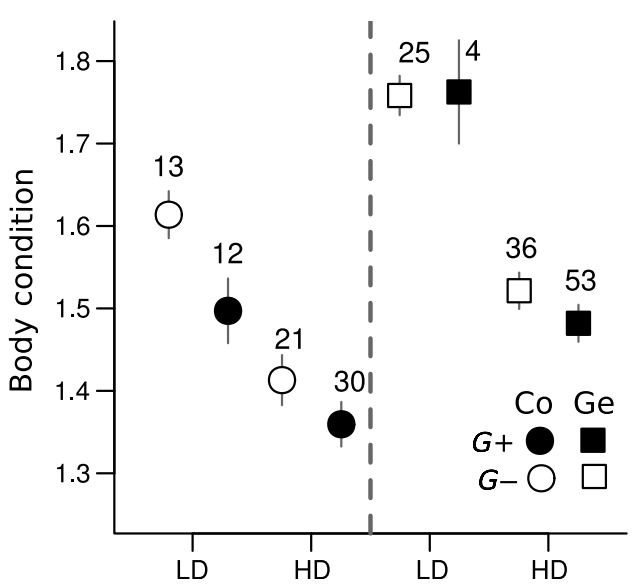

FIG. 2. Experimental design (a) and the effects on Gyrodactylus spp. on body condition (b) and (c). In (c), G+ and Gstand for the presence and absence of Gyrodactylus spp. Symbols show the experimental group means \pm standard error and numbers over symbols indicate the number of individuals in each category (see Appendix S1: Table S3 for more details in all parasites).

antihelmintic medication that reduces the prevalence of common fish parasites such as Apatemon spp., Diplostomum spp., Gyrodactylus spp., and Schistocephalus solidus and with minimal side effects on fish health and behavior (MacColl and Chapman 2010). Fish assigned to the $-I$ treatment (reduction of parasite load) were treated with $10 \mathrm{mg} / \mathrm{L}$ of Wormex dissolved in a $0.64 \% \mathrm{NaCl}$ solution, following the manufacturer's specifications. Fish assigned to the $+I$ treatment were exposed to the 
saline solution without Wormex. After a 3-h bath, all fish were transferred to clean tap water and kept in the laboratory for 2 weeks in nonflow aquaria. All individuals were completely dissected, visually inspected under an dissection scope (Leica MZ6, Wetzlar, Germany) for the presence and abundance of four focal helminth parasites (Apatemon spp., Diplostomum spp., Gyrodactylus spp., and $S$. solidus) and the protozoa, Ichthyophthirius multifiliis. All parasites were identified morphologically. For each individual, we screened the body surface and gills for Gyrodactylus spp. and I. multifiliis, then we dissected the body cavities to check for $S$. solidus. Eyes were removed and opened to check for Diplostomum spp. and Apatemon spp. cysts. This initial laboratory confirmed that Wormex treatment significantly reduced parasite load, mainly via reductions of Gyrodactylus spp. (Appendix S1: Table S1), and significantly changed the parasite community structure $\quad($ MLRT $=33.65$, $P=0.002)$.

Following this laboratory experiment, we used a similar approach to manipulate the parasite load of fish used in the mesocosm experiment. Specifically, we exposed fish intended for the $-I$ treatment to $10 \mathrm{mg} / \mathrm{L}$ Wormex, and $+I$ fish to a saline solution without Wormex. We treated 130 fish (65:65, Constance:Geneva) in nonflow aquariums, and after $3 \mathrm{~h}$ of treatment all fish were transferred to clean tap water and held for 2 weeks prior to their addition to the mesocosms.

\section{Ecosystem sampling and fish collection}

At the end of the mesocosm experiment, we measured ecosystem functions such as gross primary productivity (GPP), ecosystem respiration (ER), turbidity (attenuation of photosynthetically available light radiation, LKD), sedimentation rate (SED), and bacterial respiration (BR). These five ecosystem functions were combined into a multifunctionality index (Byrnes et al. 2014). We also sampled four main biological ecosystem properties (biomass and diversity of zooplankton, zoobenthos, phytoplankton, and periphyton). For zooplankton, $10 \mathrm{~L}$ of water was filtered through a $95 \mu \mathrm{m}$ mesh and preserved at $-20^{\circ} \mathrm{C}$ for further identification and analyses. Zoobenthos was collected from the sediment from a plastic box $(10.5 \times 10.5 \mathrm{~cm})$ preinstalled at the start of the experiment in each tank. Zooplankton and zoobenthos were identified at least to the order level, and the average biomass of each taxon was estimated by applying length-weight regressions (Appendix S1: Table S2). Phytoplankton biomass, periphyton cover, GPP, ER, LKD, SED, and BR were measured as described in Matthews et al. (2016). Two days after sampling the ecosystem, all surviving fish were collected, euthanized with an overdose of MS222, and immediately scanned for Gyrodactylus spp., I. multifiliis, Diplostomum spp., and Apatemon spp. under a dissection scope (Leica MZ6). Infections by $S$. solidus were not observed in the laboratory or the mesocosm experiments. For each fish, we measured standard body length (mm) and wet body mass ( $\mathrm{g}$ ) to obtain an estimate of individual body condition (Peig et al. 2009).

\section{Data Analyses}

\section{Parasite load and body condition}

We tested for differences in parasite load and composition using generalized multivariate models (Wang et al. 2012), with four focal parasites (e.g., Apatemon spp., Diplostomum spp., Gyrodactylus spp., and Ichthyophthirius multifiliis), and the factorial treatments and experimental block as fixed effects. For body condition, we used linear mixed effect models (LMM) with treatments and the parasite species with the strongest negative effect on body condition (Appendix S1: Fig. S1) as covariates, and tank nested within block as a random effect. Fish mortality (number of dead fish/introduced fish per tank) was modeled using generalized linear models (GLMM) with a binomial-logit error distribution.

\section{Direct and indirect ecosystem effects}

We tested the effects of fish lineage, density, and parasite load on trophic cascades and ecosystem functioning in three complementary ways. First, we tested the effects of the treatment combinations on the biomass and structure of prey communities (e.g., zooplankton and zoobenthos) with multivariate-based models, and on primary producers (e.g., phytoplankton biomass and periphyton cover) and ecosystem multifunctionality index (EMF) with LMMs using fish lineage $\times$ density $\times$ parasite load as fixed effects and block as a random effect. In the multivariate analyses, we used block as a fixed effect to control for the effects of block. Ecosystem multifunctionality was calculated by standardizing all ecosystem functions (GPP, ER, BR, SR, and LKD) using $z$ scores, and then averaging them following Byrnes et al. (2014). We used an average index to measure ecosystem functioning because we are interested in the simultaneous effect of the factorial treatments on multiple ecosystem functions, so that high values of EMF will represent high levels of ecosystem productivity (Manning et al. 2018). Overall, this analysis approach tests for treatment effects on individual and composite (e.g., EMF index) metrics, but not for covariation between metrics that would provide evidence for trophic cascades. In our experiment, evidence for trophic cascades would exist if increasing fish density both increases primary producer biomass (i.e., benthic or pelagic algae), in addition to either decreasing consumer biomass or altering consumer species composition (i.e., zooplankton or zoobenthos).

Second, in order to test for the occurrence of trophic cascades, we used natural log-response ratios (LRR) of changing fish density using a Bayesian framework by modeling the effects of lineage $\times$ density $\times$ parasite 
load then calculating the posterior distribution of predicted values for each treatment combination (Appendix S1: Supplementary methods). The effects of changing stickleback density, were then estimated as posterior probability density of $\ln \left(V_{\mathrm{HD}} / V_{\mathrm{LD}}\right)$ where $V_{\mathrm{HD}}$ and $V_{\mathrm{LD}}$ denote, respectively, the value of a variable $(V)$ at high and low- fish density. For example, the effects of stickleback density on algae were measured as $\ln \left(A_{\mathrm{HD}} /\right.$ $\left.A_{\mathrm{LD}}\right)$. In this case, we consider evidence for a trophic cascade when the posterior probability of algae LRR is significantly positive and the posterior probability of grazer LRR of either grazer biomass or composition is significantly negative. Significance is determined by whether the $95 \%$ credible intervals (CI) overlap with zero or not for both axes (Shurin et al. 2002). Using the posterior distribution from the Bayesian model allows us to estimate the posterior probability of a trophic cascade.

Third, in order to infer causal relationships between the treatments, grazer communities, primary producers, and the EMF index, we built a piecewise structural equation model (SEM, Lefcheck 2016; Appendix S1: Fig. S2). In the causal network approach, we used fish density and parasite load as exogenous variables, grazer community biomass and composition as intermediates (i.e., between fish and primary producers), and EMF as the end point. We consider evidence of trophic cascades from the SEM model when adjacent trophic modules (e.g., zooplankton and algae) are connected by significant negative effects. Piecewise SEM is a particularly useful method to test for trophic cascades because it can (1) piece together multiple (generalized) linear models into a single causal network to estimate significant indirect effects, (2) control for the effects of random structures, and (3) compare nested models, while correcting for small sample size (Lefcheck 2016). The overall fit of a piecewise SEM is tested using the Shipley test of d separation (Shipley 2009), which validates missing paths, which are not included in the model but that can improve its fit. To reject or accept a SEM model, the combined probabilities of all the paths are compared with a chi-square distribution with $2 k$ degrees of freedom (i.e., number of independent claims). If the resulting Fisher's $C$ value is unlikely to have occurred by chance $(P<0.05)$, then the SEM model is rejected because it does not have a good fit to the data (Shipley 2009, Lefcheck 2016). Additionally, we tested whether fish lineage had contrasting effects by comparing a multigroup SEM with an overall model (Shipley 2002). With this approach, we could test for the lineage effects both on the entire model and on each causal path independently (Shipley 2002).

All statistical analyses were performed in R-v3.2.3 (R Core Team 2015). To test the multivariate effects of the treatments on the structure of the parasites community, zooplankton community, zoobenthos community, and ecosystem function, we used multivariate models with the functions manlyglm or manylm from the Rpackage mvabund (Wang et al. 2012). We calculated statistical significance of each treatment with a multivariate likelihood-ratio test (MLRT) by re-sampling the data 999 times. The Bayesian models were performed using the $\mathrm{R}$ package rethinking (McElreath 2016) in STAN (Gelman et al. 2015). Models were sampled from four Hamilton Monte Carlo chains and 4,000 interactions. We used uninformative priors, for example, dnor$\operatorname{mal}(0,100)$ for all the regression prameters, except for the model variances $($ dcauchy $(0,1))$, and verified that all four chains converged using the estimated potential scale reduction statistic ( $\hat{R}$; McElreath 2016).

\section{RESULTS}

\section{Parasite community}

In the mesocosm experiment, the medication significantly reduced parasite load, which, as in the laboratory experiment, was mainly driven by a reduction of Gyrodactylus spp. These effects were stronger for the Constance than the Geneva fish (Fig. 2b). Apatemon spp. parasites were more common in Geneva fish, while and Ichthyophthirius multifiliis infections were more common on Constance fish. Fish density also had significant effects on the parasite community, mostly by increasing the densities of I. multifiliis and Gyrodactylus spp. (Appendix S1: Fig. S3).

We found that Gyrodactylus spp. $(r=-0.2, P<0.05)$ and I. multifiliis $(r=-0.15, \quad P<0.05)$ had the strongest negative correlation with fish body condition (Appendix S1: Fig. S2a). Therefore, we tested the combined effects of the experimental treatments and the presence and absence of Gyrodactylus spp. on body condition. Body condition was lower in Constance fish $\left(\mathrm{LMM}, F_{1,28.402}=31.286, P<0.001\right)$, at high fish densities (LMM, $\left.F_{1,46.93}=65.290, P<0.001\right)$, and when infected with Gyrodactylus spp. (LMM, $F_{1}, 160.179=$ 4.393, $P=0.025)$. Constance fish also had higher levels of mortality (GLMM, $X_{2,40}^{2}=10.948, P=0.01$; Mortality: Constance: $28.32 \%$ and Geneva: $11.30 \%$ ). However, because dead fish were removed and replaced, this differential mortality did not lead to sustained density and fish biomass differences between treatments. Fish mortality was not significantly influenced by the disinfection treatment (GLMM, $X_{2,40}^{2}=2.9211, P=0.232$ ). Overall, the disinfection treatment successfully reduced parasite load and changed the structure of the parasite community, and, as expected, this was associated with higher condition of stickleback in the $-I$ treatment.

Direct and indirect ecosystem effects. - At the end of the mesocosm experiment, the presence of stickleback strongly reduced zooplankton biomass, increased zoobenthos biomass, and altered the structure of the zoobenthos community (Fig. 3a, b, d). Within the factorial treatments, zooplankton biomass was marginally affected by the interaction between fish density and parasite load (Fig. 3a), and zoobenthos biomass was 
(a) Zooplankton biomass

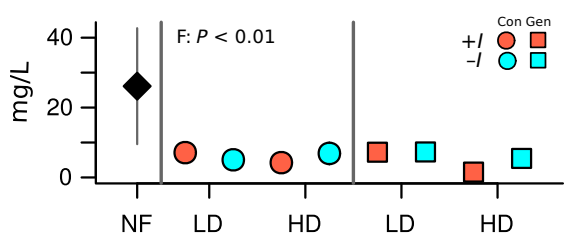

(c) Zooplankton structure

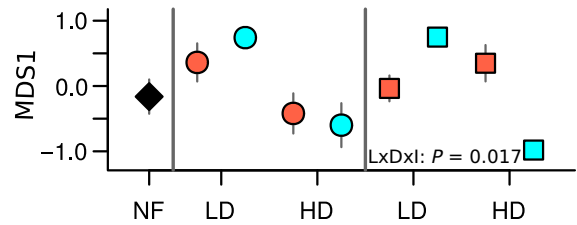

(e) Phytoplankton biomass

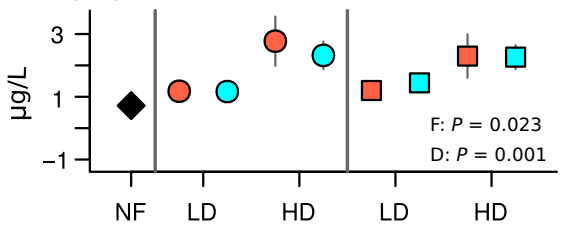

(g) Gross primary productivity (GPP)

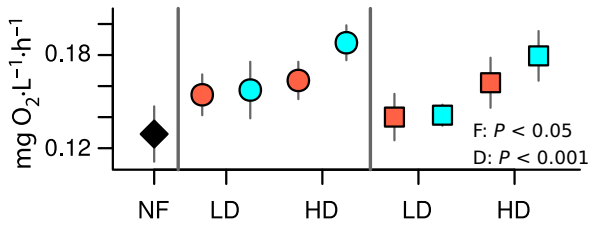

(i) Light transmission

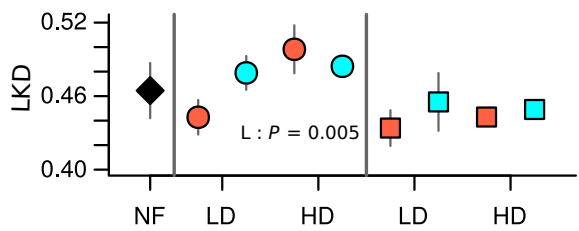

(b)Zoobenthos biomass

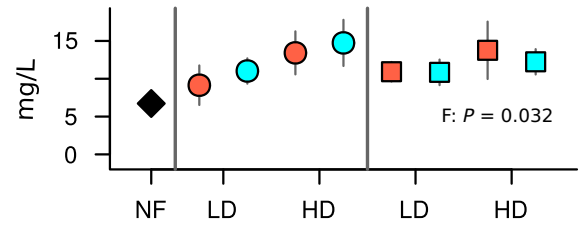

(d) Zoobenthos structure

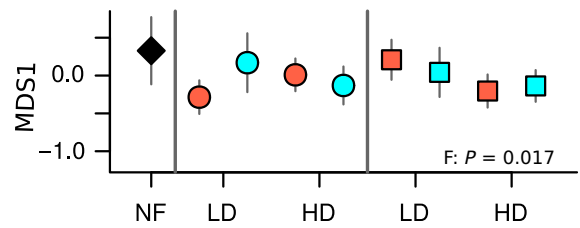

(f) Periphyton cover

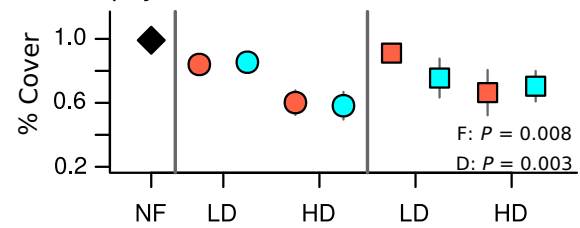

(h) Ecosystem respiration (ER)

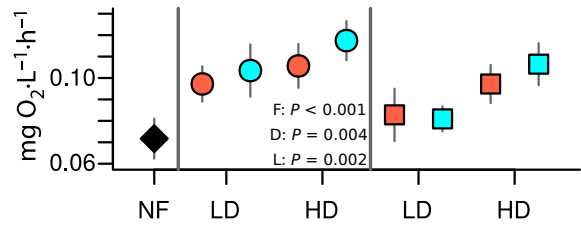

(j) Ecosystem multifunctionality

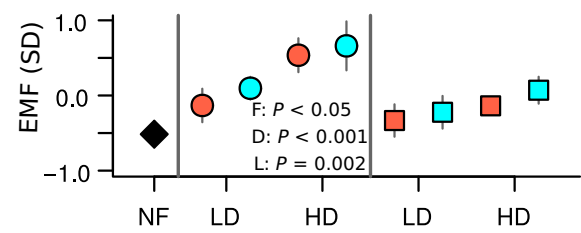

FIG. 3. Effects of stickleback treatments on ecosystem properties (a-f) and functions (g-j). Panels (a) and (b) show the effects on the zooplankton and zoobenthos biomass; panels (c) and (d), show the effects on the structure of zooplankton and zoobenthos community measured as changes in the first multi-dimensional scaling factor from a unconstrain RDA (MDS1, Appendix S1: Fig. S2); and panels (e) and (f), on the biomass of phytoplankton and the percentage cover of periphyton. Panels (g) and (h) show the stickleback effects on gross primary productivity and ecosystem respiration; and panels (i) and (j) the effects of stickleback on light transmission and ecosystem multifunctionality. Letters and numbers inside the panels highlight the statistically significant treatments e.g. fish presence (F), lineage (L), density (D), and parasite load (I). The labels of x-axis show the results for the different density treatments: no-fish (NF), low-density (LD), and high-density (HD). For more detail information see Appendix S1: Tables S2, S3 for more details. Symbols represent mean values and bars standard errors.

marginally affected by fish density (Fig. 3c). Zooplankton community structure, however, was strongly affected by the interaction of lineage, density, and parasite load treatment (Fig. 3b; Appendix S1: Table S3). Specifically, at high density, $-I$ fish had stronger negative effects on copepods $\left(F_{1,28}=8.399, P<0.01\right)$; and Geneva fish had stronger positive effects on predatory mites in high density tanks with $-I$ fish $($ Acari, lineage $\times$ density $\times$ parasite load: $F_{1,28}=7.1700, \quad P=0.012$; Appendix S1: Fig. S4 and Table S4). Unlike the zooplankton community, variation in the zoobenthos community structure and total biomass did not vary among the lineage, density, and parasite load treatment combinations (Fig. 3b, d). Furthermore, we found that copepods and mayfly larvae (Caenis) had the strongest negative correlation with phytoplankton $(r=-0.44, P<0.05)$ and periphyton $\quad(r=-0.19, \quad P<0.05 ; \quad$ Appendix $\quad \mathrm{S} 1$ : Fig. S2b, c). Overall, increasing stickleback density increased ecosystem productivity (Fig. 3e-j). At high fish density, mesocosms had more phytoplankton, less periphyton, and higher levels of GPP, ER, and EMF than low density tanks (Appendix S1: Table S4). 
We also found that mesocosms with Constance fish had higher levels of ER, LKD, and EMF (Appendix S1: Table S4).

We tested for trophic cascades mediated by either changes in grazer biomass (DMIE) or community composition, which could ultimately impact grazer feeding rates and/or be the results of changes in fish behavior (e.g., TMIE). In both cases, there was stronger evidence for a pelagic trophic cascade than for a benthic trophic cascade when increasing fish density (Table 1, Fig. 4). The Bayesian LRR (Fig. 4) and the structural equation model (SEM, Fig. 5a) both revealed that increasing stickleback density strongly impacted zooplankton community composition (MDS1), and this was associated associated with higher phytoplankton biomass and EMF (path: HD $\rightarrow$ Zoo MDS1 $\rightarrow$ Phytoplankton $\rightarrow$ EMF; Fig. 5).

The Bayesian LRR additionally revealed that the probability of trophic cascades was very similar for both lineages (Table 1). Consistent with this, we found that Constance and Geneva stickleback did not differentially affect the overall structure of the SEM model (i.e., a multi group SEM was not supported: $X_{1,36}^{2}=49.461$, $P=0.066$ ). However, in the overall SEM, we did find lineage-specific differences in parameter estimates relating to pathways underlying trophic cascades (Fig. 5b). These pathways include both the effect of fish density on zooplankton community structure (Fig. 5b), which was $21.6 \%$ stronger for Geneva fish $\left(X_{1,95}^{2}=36.139\right.$, $P<0.01)$ and the effect of zooplankton community structure on algal biomass (Fig. 5b), which was $23.2 \%$ stronger for Constance stickleback $\left(X_{1,95}^{2}=8.93\right.$, $P<0.01$, Appendix S1: Table S7).

Overall, reducing parasite load increased the probability of detecting pelagic trophic cascades by more than $30 \%$, especially for cascading effects mediated by changes in the zooplankton community structure (Table 1 and Fig. 4). This outcome is also consistent with a non-Bayesian LRR analysis (Appendix S1: Fig. S6), and the SEM modeling (Fig. 5a). Whereas the overall effects of a high fish density led to a large shift in zooplankton composition (red arrow with coefficient
$-0.74)$, a high density of fish with natural parasite infections levels $(+I)$ showed a much weaker impact on zooplankton composition (black arrow with coefficient 0.64 counteracts these negative effects). The weaker effects of fish with natural parasite loads on zooplankton translated into weaker indirect effects on phytoplankton biomass (path: HD: $+I \rightarrow$ Zoo MDS1 $\rightarrow$ Phytoplankton, Fig. 5a).

\section{Discussion}

Overall, our experiment tests how parasites can influence the top-down effects of predators on trophic cascades and ecosystem functioning. Specifically, we found that increasing stickleback density caused trophic cascades in the pelagic but not the benthic food chain (Fig. 4). Variation in periphyton cover was also affected by fish density, but we found no evidence linking it to changes in zoobenthos biomass or composition (Fig. 5). Pelagic trophic cascades occurred because of effects of stickleback density and parasite load on zooplankton community composition rather than zooplankton biomass (Table 1). Evidence of trophic cascades was stronger when parasite load of fish was reduced (Figs. 4, 5a, Table 1) and for the population with the more pelagic phenotype (i.e., Lake Constance: Fig. 5b). The index of EMF, showed stronger support for lineage and density effects than for parasite effects (Fig. 5; Appendix S1: Fig. S5), but a large portion of the variation (86\%) in EMF remains unexplained in the SEM model.

Although parasites are well known to affect the physiology and behavior of hosts (Barber and Dingemanse 2010), little is known about the reach of their effects across multiple trophic levels (Wood and Johnson 2015). Parasites directly influence zooplankton community dynamics (Duffy 2007), zoobenthos (Mouritsen and Haun 2008) and primary producers (Ibelings et al. 2004); and some parasites with complex life cycles can even affect multiple trophic levels at different stages of their life cycle (Lafferty and Kuris 2012). Here, we found that reductions of parasite load (of multiple parasite species) increase likelihood of stickleback-mediated trophic

TABLE 1. Posterior probability (\%) of detecting density mediated trophic cascades given stickleback lineage and parasite load.

\begin{tabular}{|c|c|c|c|c|c|c|}
\hline & \multicolumn{3}{|c|}{ Grazer's biomass } & \multicolumn{3}{|c|}{ Grazer's structure } \\
\hline & Constance & Geneva & Parasite load & Constance & Geneva & Parasite load \\
\hline \multicolumn{7}{|c|}{ Pelagic trophic cascades $(\%)$} \\
\hline$+I$ & 50.05 & 50.30 & 50.04 & 51.22 & 50.75 & 51.79 \\
\hline$-I$ & 75.18 & 83.54 & 83.23 & 99.13 & 96.91 & 99.61 \\
\hline Lineage & 54.40 & 50.67 & & 52.09 & 51.6 & \\
\hline \multicolumn{7}{|c|}{ Benthic trophic cascade $(\%)$} \\
\hline$+I$ & 48.93 & 49.19 & 48.93 & 49.56 & 43.44 & 49.59 \\
\hline$-I$ & 7.88 & 30.25 & 10.88 & 29.52 & 23.52 & 21.08 \\
\hline Lineage & 48.47 & 48.98 & & 49.325 & 49.33 & \\
\hline
\end{tabular}

Notes: Pelagic trophic cascades were more likely in mesocosms with $-I$ fish $(>83 \%)$. The probability of detecting benthic trophic cascades was $<50 \%$ for any treatment. 
(a)

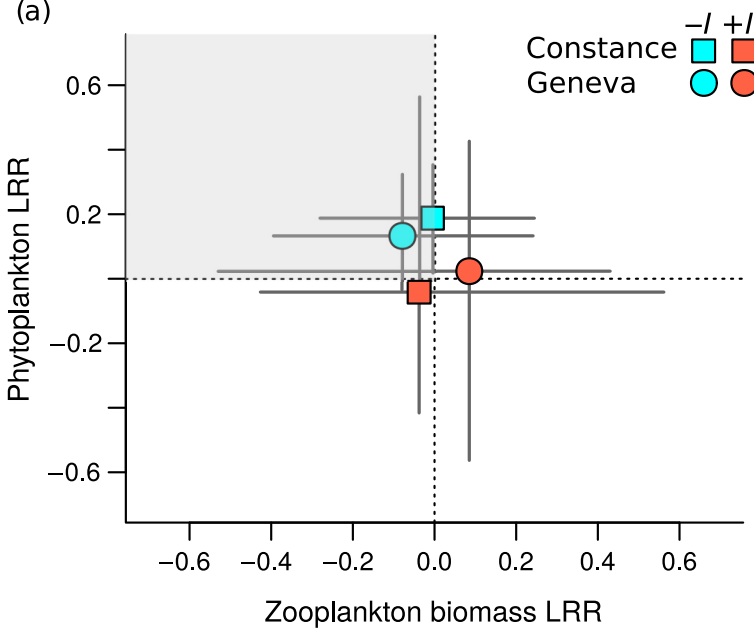

(c)

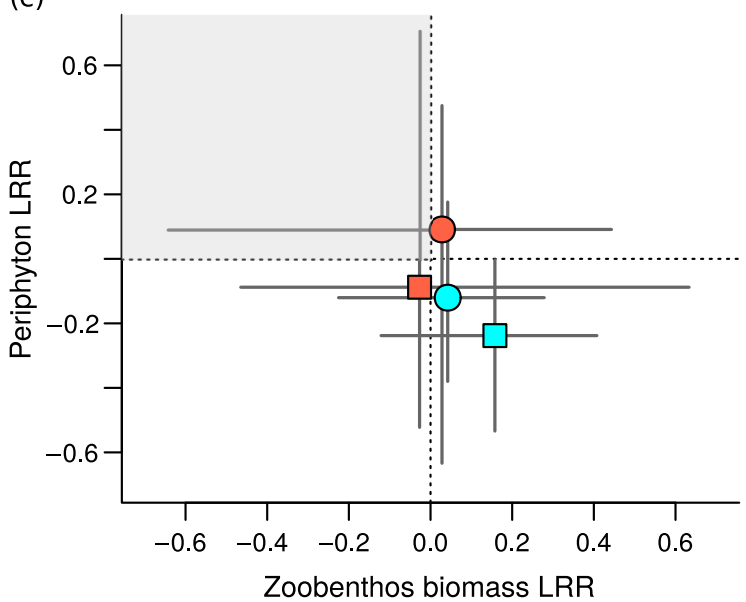

(b)

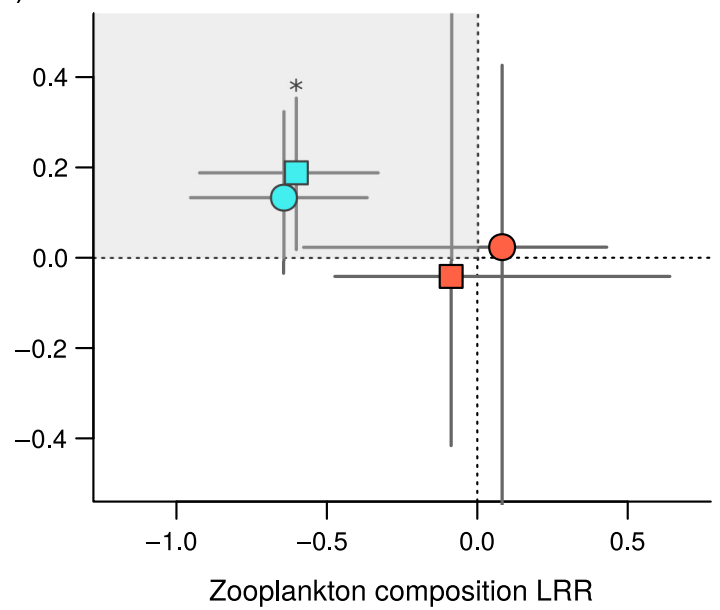

(d)

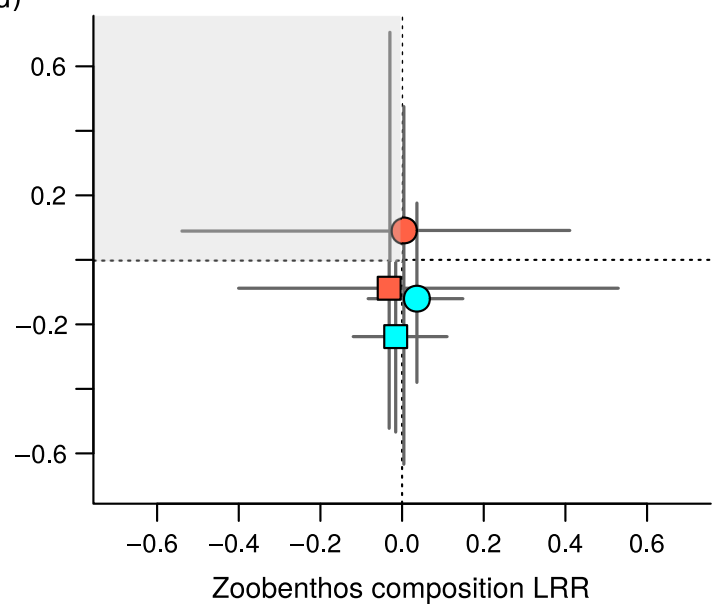

FIG. 4. Relationship between the direct effects ( $x$-axes) of stickleback on grazers (pelagic: (a) and (b); benthic: (c) and (d)) and the indirect effects ( $y$-axes) on algae (pelagic: (a) and (b); benthic: (c) and (d)). Symbols show the net effect of changes in fish density given stickleback lineage and parasite load. There is evidence of significant trophic cascading effects when the point estimate of the effect (mode from the posterior distribution) magnitudes are in the gray area and the $95 \%$ credible intervals are not overlapping with the zero line (Shurin et al. 2002). Significant trophic cascades are highlighted with an asterisk. For more details on the Bayesian models see Appendix S1: Table S6. LRR, log-response ratios.

cascades in the pelagic environment (Fig. 5a). Parasites such as Diplostomum spp. and Apatemon spp., which can impair fish vision, might have influenced the experimental fish, but we did not find strong evidence of their deleterious effects. Instead, our results suggest that Gyrodactylus spp. and Ichthyophthirius multifiliis reduced the condition of stickleback (Eizaguirre et al. 2009, 2012), potentially altering foraging performance and behavior and leading to cascading effects on primary producers (Anaya-Rojas et al. 2016, Brunner et al. 2017).

Although many traits of prey (e.g., induced defenses, antipredator avoidance behaviors) are well known to play important roles in trophic cascades (Schmitz et al. 2004), much less is known about the importance of predator traits (Lima 2002, Abrams 2007), particularly those underlying foraging performance. Predator traits such as foraging activity (Abrams 1982) and diet choice (Ma et al. 2003) could be particularly susceptible to parasite infections due to behavioral and physiological trade-offs (Hatcher and Dunn 2011). This is consistent with our observations that the reduction of parasite load affected prey community composition but not overall biomass, and these changes led to an increased on algal biomass. Such trophic cascades have been previously dubbed cryptic, because they are difficult to detect without careful quantification of changes in community composition (Tessier and Woodruff 2002). Indeed, it is possible that the positive effect of fish on periphyton biomass (Fig. 5a) might reflect undetected changes either in the species or trait distributions of the benthic community, or in the physical and chemical environment (e.g., habitat modification, nutrient cycling). 


\section{(a) Overall model}

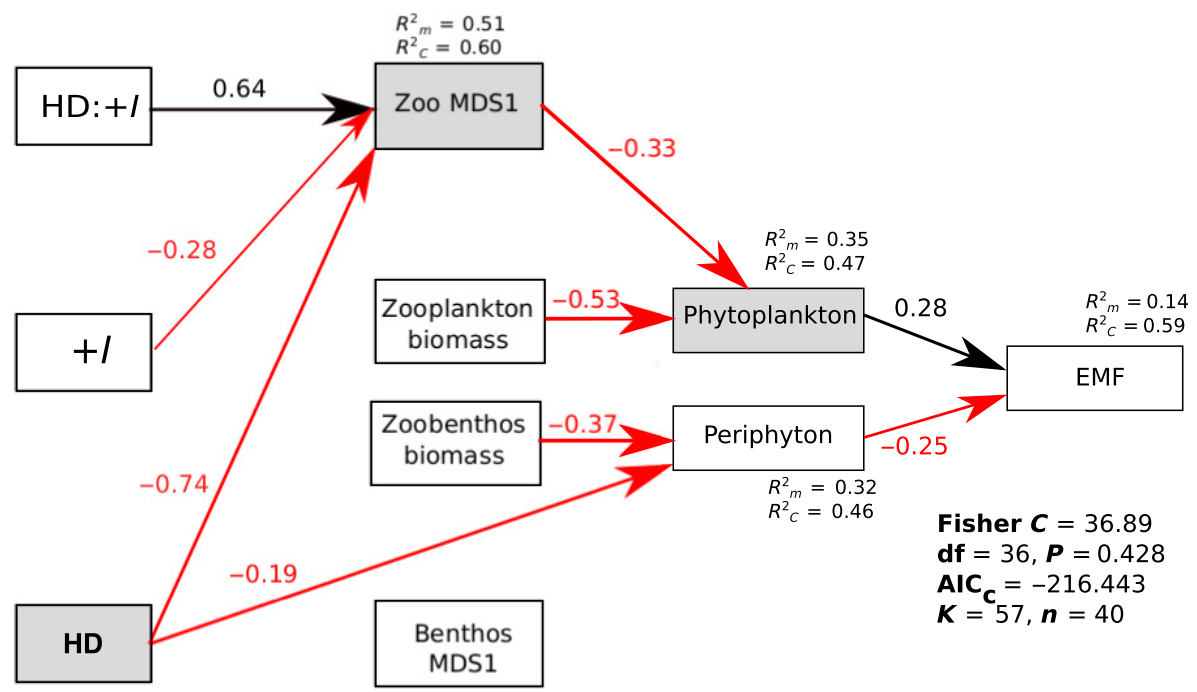

(b) Overall model showing lineage differences

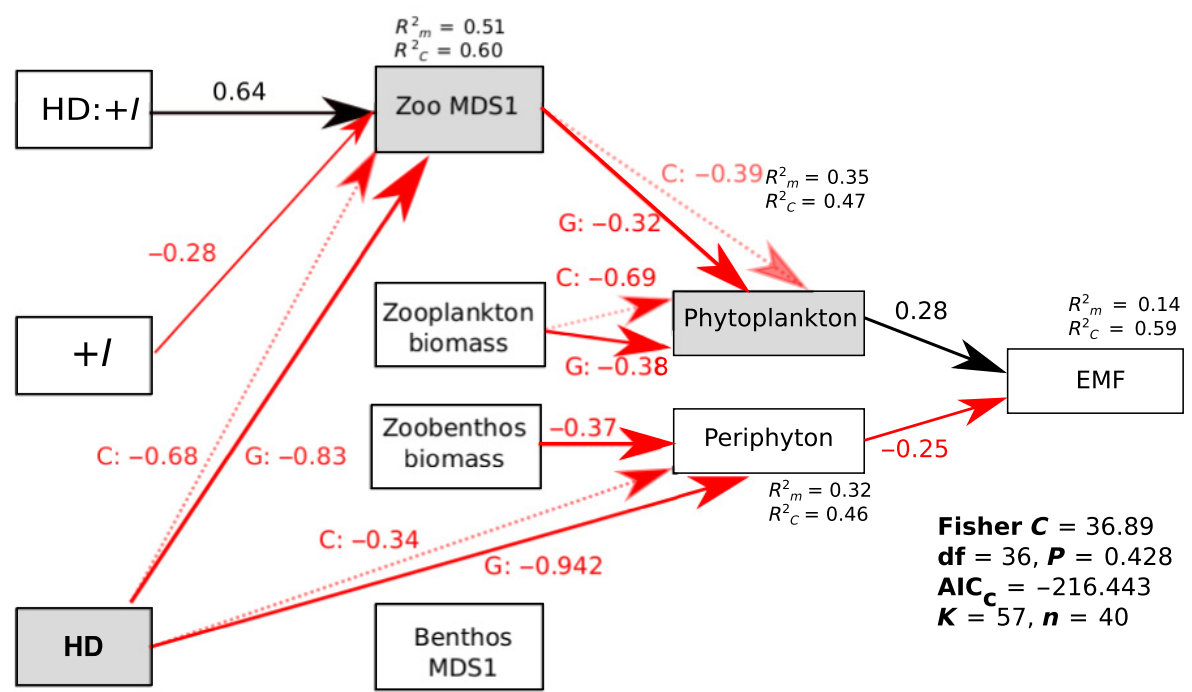

FIG. 5. Results of the structural equation model. Diagram (a) shows the best overall model, and diagram (b) shows the same model but highlighting the lineage differences in path coefficients. Here we only present significant path coefficients representing positive (black) and negative (red) effects (but see Appendix S1: Tables S5, S7 for more details). Trophic modules involved in significant trophic interactions are shaded. Numbers indicate standardized effect size ( -1 to 1$)$ of the path coefficients. Zoo MDS1 and benthos MDS1 represent the first multidimensional scaling of the zooplankton and zoobenthos community structure. The bottom right corner shows the model fitting the data for the best model $(P>0.05)$. As exogenous variables we modeled the effects of high fish density (HD) and natural infection levels $(+I)$.

Trophic cascades involving changes in community composition can be both common and strong in natural populations (Tessier and Woodruff 2002, Schmitz 2006). Factors such as predator or herbivore foraging efficiency (e.g., numerical dynamics and functional response) and community regulation (e.g., compensatory effects), for instance, can increase the strength of cryptic trophic cascades via changes in consumer conversion efficiency even without changes in the total community biomass (Pace 1984, Borer et al. 2005). In our experiment, it is likely that stickleback at high densities reduced large and efficient zooplankton grazers, without reducing the total biomass of zooplankton. It is also possible that the observed changes in zooplankton community structure influenced the structure of the phytoplankton and microbial community, which has been observed in 
previous mesocosm experiments (Birtel and Matthews 2016). Additional quantification of these communities might have improved the explanatory power of the pathways identified by the SEM analysis, and helped identify additional pathways linking fish effects with changes in ecosystem functioning (Fig. 5b).

Overall, our analyses suggest that stickleback from the Lake Constance and Lake Geneva indirectly modified mesocosm ecosystems through similar ecological mechanisms, specifically via pelagic trophic cascades mediated by changes in zooplankton community structure. However, in the more detailed analysis of SEM pathways, we found some evidence that the two lineages had differential effects on pelagic trophic cascades and on periphyton (Fig. 5b). For example, differences in path coefficients between lineages suggest that fish from Constance caused stronger pelagic trophic cascades. Specifically, the net effect of Constance stickleback density on phytoplankton biomass was greater (i.e., more positive), relative to their effect on zooplankton composition (Fig. 5b). It is unclear why fish from both lineages had strong density effects on copepods, but this only translated into a significant change in phytoplankton biomass in the Constance treatment (Appendix S1: Table S7).

Previous work suggests that Lake Geneva sticklebacks are more adapted to foraging in benthic environments, whereas Lake Constance stickleback are more adapted to pelagic environments (Lucek et al. 2013, 2014, Best et al. 2017). For instance, stickleback from Lake Constance have a more pelagic phenotype (Lucek et al. 2013) and have a higher feeding efficiency on plankton (Best et al. 2017) than stickleback from Lake Geneva. One possibility is that Constance fish had a stronger impact on phytoplankton, because they fed more selectively on more efficient grazer, but a remaining challenge is to identify which of the traits that differ between lineages are responsible for their differential community and ecosystem effects (Best et al. 2017). Lineage differences in the effect on periphyton cover could also suggest a role for nontrophic effects of stickleback on ecosystem functioning (Atkinson et al. 2017), suggesting further work is needed on mechanisms such as habitat modification and nutrient cycling (Best et al. 2017, Leal et al. 2017).

There are some limitations to our experiment that prevent us from making stronger and more general conclusions about the effects of parasite load and evolutionary lineage of stickleback on aquatic ecosystems. First, because the parasite manipulations were performed on wild-caught fish, a combination of phenotypic plasticity and genetic differences are likely to explain some variation in the effects of fish on grazer communities and ecosystem functioning (Lundsgaard-Hansen et al. 2014, Brunner et al. 2017). However, our results show that independently of the origin of the effect, stickleback parasites can affect trophic cascades. Second, replacing fish that died during the experiment with fish from the lab was essential to maintain the density contrast throughout the experiment, but it also meant that fish in higher condition from the lab were substituted into the experiment on multiple occasions. However, becuase parasites such as Gyrodactylus spp. and I. multifiliis can be easily transmitted among fish, it is likely that replacement fish would have been exposed and infected, maintaining the indirect effects of parasites on the system (Eizaguirre et al. 2009).

In this study, we tested how parasites at the top of food chains can indirectly influence grazer communities and ecosystem functioning. Such cascading effects are likely to be influenced by co-evolutionary interactions between host and parasites that vary with the environmental context (Thompson 2013, Brunner et al. 2017). Overall, our results suggest that parasites can affect ecosystems via a combination of both DMIE and TMIE, mediated by host condition and behavior of the predator. This highlights the importance of adopting both ecological and (co)evolutionary perspectives for studying the effects of species interactions on community structure and ecosystem processes.

\section{ACKNOWLEDGMENTS}

We thank D. Steiner, D. Hohmann, A. Taverna, M. Lürig, G. Antoniazza, L. Catalano, J. Naef, and B. Kienholz for their assistance in the lab, in the mesocosm garden, and in the field. We are very grateful for the support of the whole EAWAG Kastanienbaum community, especially to David Marquez, Kay Lucek, M. McGee, and two anonymous reviewers for their comments. This project was funded through the Lead Agency Project of the German Science Foundation (DFG, EI841/4-1) and the Swiss National Science Foundation (SNSF 139326) to $\mathrm{CE}$ and $\mathrm{BM}$. The project was enabled by the stickleback cluster of the DFG priority program 1399 "Host-Parasite Co-evolution" and supported by a DFG grant to CE (EI 841/6-1). JMA-R analyzed the data and wrote the first drafts of the manuscript. JMA-R, RJB, and FSB performed the experiment. MCL contributed to data collection and writing the manuscript. JMA-R, RJB, BM, CE, and OS conceived the experiment and contributed substantially to the revisions of the manuscript. CJM made substantial contributions to the model construction, structure and revisions of the manuscript draft. Here we confirm that we do not have any conflict of interest.

\section{Literature Cited}

Abrams, P. A. 1982. Functional responses of optimal foragers. American Naturalist 120:382-390.

Abrams, P. A. 1995. Implications of dynamically variable traits for identifying, classifying, and measuring direct and indirect effects in ecological communities. American Naturalist 146:112-134.

Abrams, P. A. 2007. Defining and measuring the impact of dynamic traits on interspecific interactions. Ecology 88:25552562.

Anaya-Rojas, J. M., F. S. Brunner, N. Sommer, O. Seehausen, C. Eizaguirre, and B. Matthews. 2016. The association of feeding behaviour with the resistance and tolerance to parasites in recently diverged sticklebacks. Journal of Evolutionary Biology 29:2157-2167. 
Atkinson, C. L., K. A. Capps, A. T. Rugenski, and M. J. Vanni. 2017. Consumer-driven nutrient dynamics in freshwater ecosystems: from individuals to ecosystems. Biological Reviews 92:2003-2023.

Barber, I. 2013. Sticklebacks as model hosts in ecological and evolutionary parasitology. Trends in Parasitology 29:556-566.

Barber, I., and N. J. Dingemanse. 2010. Parasitism and the evolutionary ecology of animal personality. Philosophical Transactions of the Royal Society B 365:4077-4088.

Best, R. J., J. M. Anaya-Rojas, M. C. Leal, D. W. Schmid, O. Seehausen, and B. Matthews. 2017. Transgenerational selection driven by divergent ecological impacts of hybridizing lineages. Nature Ecology and Evolution 1:1757-1765.

Birtel, J., and B. Matthews. 2016. Grazers structure the bacterial and algal diversity of aquatic metacommunities. Ecology 97:3472-3484.

Borer, E. T., E. W. Seabloom, J. B. Shurin, K. E. Anderson, C. A. Blanchette, B. Broitman, S. D. Cooper, and B. S. Halpern. 2005. What determines the strength of a trophic cascade? Ecology 86:528-537.

Brunner, F. S., J. M. Anaya-Rojas, B. Matthews, and C. Eizaguirre. 2017. Experimental evidence that parasites drive eco-evolutionary feedbacks. Proceedings of the National Academy of Sciences USA 114:3678-3683.

Buck, J. C., and W. J. Ripple. 2017. Infectious agents trigger trophic cascades. Trends in Ecology and Evolution 32:681-694.

Byrnes, J. E. K., G. Lars, I. Forest, J. S. Lefcheck, J. N. Griffin, H. Andy, B. J. Cardinale, D. U. Hooper, L. E. Dee, and J. Emmett Duffy. 2014. Investigating the relationship between biodiversity and ecosystem multifunctionality: challenges and solutions. Methods in Ecology and Evolution 5:111-124.

Duffy, M. A. 2007. Selective predation, parasitism, and trophic cascades in a bluegill-Daphnia-parasite system. Oecologia 153:453-460.

Duffy, M. A., J. H. Ochs, R. M. Penczykowski, D. J. Civitello, C. A. Klausmeier, and S. R. Hall. 2012. Ecological context influences epidemic size and parasite-driven evolution. Science 335:1636-1638.

Eizaguirre, C., T. L. Lenz, M. Kalbe, and M. Milinski. 2012. Divergent selection on locally adapted major histocompatibility complex immune genes experimentally proven in the field. Ecology Letters 15:723-731.

Eizaguirre, C., T. L. Lenz, R. D. Sommerfeld, C. Harrod, M. Kalbe, and M. Milinski. 2011. Parasite diversity, patterns of MHC II variation and olfactory based mate choice in diverging three-spined stickleback ecotypes. Evolutionary Ecology 25:605-622.

Eizaguirre, C., S. E. Yeates, T. L. Lenz, M. Kalbe, and M. Milinski. 2009. MHC-based mate choice combines good genes and maintenance of MHC polymorphism. Molecular Ecology 18:3316-3329.

Fang, B., J. Merilä, F. Ribeiro, C. M. Alexandre, and P. Momigliano. 2018. Worldwide phylogeny of three-spined sticklebacks. Molecular Phylogenetics and Evolution 127:613-625.

Gelman, A., D. Lee, and J. Guo. 2015. Stan: a probabilistic programming language for Bayesian inference and optimization. Journal of Educational and Behavioral Statistics 40:530-543.

Hatcher, M. J., J. T. A. Dick, and A. M. Dunn. 2006. How parasites affect interactions between competitors and predators. Ecology Letters 9:1253-1271.

Hatcher, M. J., and A. M. Dunn. 2011. Parasites in ecological communities. Cambridge University Press, Cambridge, UK.

Hudson, P. J., A. P. Dobson, and D. Newborn. 1998. Prevention of population cycles by parasite removal. Science 282:22562258.

Ibelings, B. W., A. De Bruin, M. Kagami, M. Rijkeboer, M. Brehm, and E. V. Donk. 2004. Host parasite interactions between freshwater phytoplankton and chytrid fungi (Chytridiomycota). Journal of Phycology 40:437-453.

Karvonen, A., K. Lucek, D. A. Marques, and O. Seehausen. 2015. Divergent macroparasite infections in parapatric Swiss lake-stream pairs of threespine stickleback (Gasterosteus aculeatus). PLoS ONE 10:e0130579.

Lafferty, K. D. 2006. Parasites dominate food web links. Proceedings of the National Academy of Sciences USA 103:11211-11216.

Lafferty, K. D., and A. M. Kuris. 2012. Ecological consequences of manipulative parasites. Pages $158-171$ in D. Hughes, J. Brodeur, and F. Thomas, editors. Host manipulation by parasites. Oxford University Press, Oxford, UK.

Leal, M. C., O. Seehausen, and B. Matthews. 2017. The ecology and evolution of stoichiometric phenotypes. Trends in Ecology and Evolution 32:108-117.

Lefcheck, J. S. 2016. piecewiseSEM: piecewise structural equation modelling in $\mathrm{r}$ for ecology, evolution, and systematics. Methods in Ecology and Evolution 7:573-579.

Lefèvre, T., C. Lebarbenchon, M. Gauthier-Clerc, D. Missé, R. Poulin, and F. Thomas. 2009. The ecological significance of manipulative parasites. Trends in Ecology and Evolution 24:41-48.

Lima, S. L. 2002. Putting predators back into behavioral predator-prey interactions. Trends in Ecology and Evolution 17:70-75.

Lucek, K., D. Roy, E. Bezault, A. Sivasundar, and O. Seehausen. 2010. Hybridization between distant lineages increases adaptive variation during a biological invasion: stickleback in Switzerland. Molecular Ecology 19:39954011.

Lucek, K., A. Sivasundar, B. K. Kristjánsson, S. Skúlason, and O. Seehausen. 2014. Quick divergence but slow convergence during ecotype formation in lake and stream stickleback pairs of variable age. Journal of Evolutionary Biology 27:1878-1892.

Lucek, K., A. Sivasundar, D. Roy, and O. Seehausen. 2013. Repeated and predictable patterns of ecotypic differentiation during a biological invasion: lake-stream divergence in parapatric Swiss stickleback. Journal of Evolutionary Biology 26:2691-2709.

Lundsgaard-Hansen, B., B. Matthews, and O. Seehausen. 2014. Ecological speciation and phenotypic plasticity affect ecosystems. Ecology 95:2723-2735.

Ma, B. O., P. A. Abrams, and C. E. Brassil. 2003. Dynamic versus instantaneous models of diet choice. American Naturalist 162:668-684.

MacColl, A. D. C., and S. M. Chapman. 2010. Parasites can cause selection against migrants following dispersal between environments. Functional Ecology 24:847-856.

Manning, P., F. Plas, S. Soliveres, E. Allan, F. T. Maestre, G. Mace, M. J. Whittingham, and M. Fischer. 2018. Redefining ecosystem multifunctionality. Nature Ecology \& Evolution 2:427.

Matthews, B., T. Aebischer, K. E. Sullam, B. Lundsgaard-Hansen, and O. Seehausen. 2016. Experimental evidence of an eco-evolutionary feedback during adaptive divergence. Current Biology 26:483-489.

McElreath, R. 2016. Statistical rethinking: a Bayesian course with examples in R and Stan. CRC Press, Boca Raton, Florida, USA.

Milinski, M. 1984. Parasites determine a predator's optimal feeding strategy. Behavioral Ecology and Sociobiology 15:35-37.

Mouritsen, K. N., and S. C. B. Haun. 2008. Community regulation by herbivore parasitism and density: trait-mediated indirect interactions in the intertidal. Journal of Experimental Marine Biology and Ecology 367:236-246. 
Pace, M. L. 1984. Zooplankton community structure, but not biomass, influences the phosphorus-chlorophyll a relationship. Canadian Journal of Fisheries and Aquatic Sciences 41:1089-1096.

Peig, J., P. Jordi, and A. J. Green. 2009. New perspectives for estimating body condition from mass/length data: the scaled mass index as an alternative method. Oikos 118:1883-1891.

Polis, G. A., A. L. W. Sears, G. R. Huxel, D. R. Strong, and J. Maron. 2000. When is a trophic cascade a trophic cascade? Trends in Ecology and Evolution 15:473-475.

Post, D. M., E. P. Palkovacs, E. G. Schielke, and S. I. Dodson. 2008. Intraspecific variation in a predator affects community structure and cascading trophic interactions. Ecology 89:2019-2032.

R Core Team. 2015. R: a language and environment for statistical computing. R Foundation for Statistical Computing, Vienna, Austria. www.r-project.org

Ripple, W. J., J. A. Estes, O. J. Schmitz, V. Constant, M. J. Kaylor, A. Lenz, J. L. Motley, K. E. Self, D. S. Taylor, and C. Wolf. 2016. What is a trophic cascade? Trends in Ecology and Evolution 31:842-849.

Rudman, S. M., J. Heavyside, D. J. Rennison, and D. Schluter. 2016. Piscivore addition causes a trophic cascade within and across ecosystem boundaries. Oikos 125:1782-1789.

Schmitz, O. J. 2006. Predators have large effects on ecosystem properties by changing plant diversity, not plant biomass Ecology 87:1432-1437.

Schmitz, O. J. 2008. Effects of predator hunting mode on grassland ecosystem function. Science 319:952-954.

Schmitz, O. J., V. Krivan, and O. Ovadia. 2004. Trophic cascades: the primacy of trait-mediated indirect interactions. Ecology Letters 7:153-163.
Seppälä, O., A. Karvonen, and E. Valtonen. 2011. Eye fluke-induced cataracts in natural fish populations: Is there potential for host manipulation? Parasitology 138:209-214.

Shipley, B. 2002. Cause and correlation in biology: a user's guide to path analysis, structural equations and causal inference. Cambridge University Press, Cambridge, UK.

Shipley, B. 2009. Confirmatory path analysis in a generalized multilevel context. Ecology 90:363-368.

Shurin, J. B., E. T. Borer, E. W. Seabloom, K. Anderson, C. A Blanchette, B. Broitman, S. D. Cooper, and B. S. Halpern. 2002. A cross-ecosystem comparison of the strength of trophic cascades: strength of cascades. Ecology Letters 5:785-791.

Start, D., and B. Gilbert. 2017. Predator personality structures prey communities and trophic cascades. Ecology Letters $20: 366-374$

Tessier, A. J., and P. Woodruff. 2002. Cryptic trophic cascade along a gradient of lake size. Ecology 83:1263-1270.

Thompson, J. N. 2013. Relentless evolution. University of Chicago Press, Chicago, Illinois, USA.

Wang, Y., U. Naumann, S. T. Wright, and D. I. Warton. 2012. mvabund - an R package for modelbased analysis of multivariate abundance data. Methods in Ecology and Evolution $3: 471-474$.

Wolinska, J., and K. C. King. 2009. Environment can alter selection in host-parasite interactions. Trends in Parasitology 25:236-244.

Wood, C. L., and P. T. J. Johnson. 2015. A world without parasites: exploring the hidden ecology of infection. Frontiers in Ecology and the Environment 13:425-434.

\section{SUPPORTING INFORMATION}

Additional supporting information may be found in the online version of this article at http://onlinelibrary.wiley.com/doi/ 10.1002/ecy.2744/suppinfo

\section{Data Availability}

